Article

\title{
Genome-Wide Identification and Expression Analysis of the NRAMP Family Genes in Tea Plant (Camellia sinensis)
}

\author{
Jinqiu Li ${ }^{1}$, Yu Duan ${ }^{1}$, Zhaolan Han ${ }^{1}$, Xiaowen Shang ${ }^{1}$, Kexin Zhang ${ }^{1}$, Zhongwei Zou ${ }^{2} \mathbb{1}$, Yuanchun Ma ${ }^{1}$, \\ Fang $\mathrm{Li}^{1}$, Wanping Fang ${ }^{1}$ and Xujun $\mathrm{Zhu}{ }^{1, *}$ (D) \\ 1 College of Horticulture, Nanjing Agricultural University, Nanjing 210095, China;

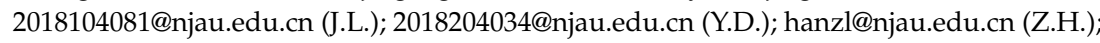 \\ 2019104081@njau.edu.cn (X.S.); 2019104086@njau.edu.cn (K.Z.); myc@njau.edu.cn (Y.M.); \\ lifang@njau.edu.cn (F.L.); fangwp@njau.edu.cn (W.F.) \\ 2 Department of Plant Science, University of Manitoba, 66 Dafoe Road, Winnipeg, MB R3T 2N2, Canada; \\ Zhongwei.Zou@umanitoba.ca \\ * Correspondence: zhuxujun@njau.edu.cn; Tel./Fax: +86-25-8439-5182
}

Citation: Li, J.; Duan, Y.; Han, Z.; Shang, X.; Zhang, K.; Zou, Z.; Ma, Y.; Li, F.; Fang, W.; Zhu, X. Genome-Wide Identification and Expression Analysis of the NRAMP Family Genes in Tea Plant (Camellia sinensis). Plants 2021, 10, 1055. https:// doi.org/10.3390/plants10061055

Academic Editor: Anja Schneider

Received: 29 April 2021

Accepted: 21 May 2021

Published: 25 May 2021

Publisher's Note: MDPI stays neutral with regard to jurisdictional claims in published maps and institutional affiliations.

Copyright: (C) 2021 by the authors Licensee MDPI, Basel, Switzerland. This article is an open access article distributed under the terms and conditions of the Creative Commons Attribution (CC BY) license (https:// creativecommons.org/licenses/by/ $4.0 /)$.

\begin{abstract}
The natural resistant-associated macrophage protein (NRAMP) is a kind of integral membrane transporter which could function on a wide range of divalent metal ions in plants. Little is known about the NRAMP family in Camellia sinensis. In this study, 11 NRAMP genes were identified from the tea plant genome. Phylogenetic analysis showed that the 11 CsNRAMP proteins were split into two groups. The proteins of group 1 contained the conserved motif 6 (GQSSTxTG), while most proteins in group 2 (excepting CsNRAMP7 and CsNRAMP10) contained the conserved residues of motif 6 and motif 2 (GQFIMxGFLxLxxKKW). The number of amino acids in coding regions of 11 CsNRAMP genes ranged from 279-1373, and they contained 3-12 transmembrane domains. Quantitative RT-PCR analysis showed that G1 genes, CsNRAMP3, CsNRAMP4, and CsNRAMP5, were extraordinarily expressed in roots, while G2 genes showed higher expression levels in the stems and leaves. The expression levels of CsNRAMPs in roots and leaves were detected to assess their responses to $\mathrm{Pb}$ treatment. The results indicated that CSNRAMPs were differentially regulated, and they might play a role in $\mathrm{Pb}$ transportation of tea plant. Subcellular localization assay demonstrated that CsNRAMP2 and CsNRAMP5 fused proteins were localized in the plasma membrane. Overall, this systematic analysis of the CSNRAMP family could provide primary information for further studies on the functional roles of CSNRAMPs in divalent metal transportation in tea plants.
\end{abstract}

Keywords: Camellia sinensis; $N R A M P$; $\mathrm{Pb}$ treatment; gene expression

\section{Introduction}

As one of the most popular nonalcoholic beverage crops, the tea plant has been widely cultivated throughout the world [1]. The tea plant can absorb various nutrients and essential elements from soil to maintain growth and development [2]. However, some toxic substances, such as $\mathrm{Pb}$, are also accumulated, which could have adverse impacts on morphological, physiological, and biochemical properties in plants [3,4]. Lead, as one of the toxic heavy metals, could block the function of essential metals and induce the production of ROS [5]. It could destroy the electron transport chain and induce lipid peroxidation [6]. Furthermore, toxic effects may appear in other cell mechanisms, such as water balance, protein structure, and photosynthesis [7,8]. Additionally, $24.4 \%$ to $72 \%$ of the total lead content in the dried black tea could be released into the tea infusion [9]. In a 74-sample study, $17.57 \%$ of the samples had a higher concentration of $\mathrm{Pb}$ than the maximum limits in tea leaves [10]. Thus, $\mathrm{Pb}$ pollution could cause significant damage to the tea plant, and even be harmful for consumer health [11]. Due to the rapid development of modern industry, urban activities, and transportation, lead contamination in tea has become a major 
concern [12-14]. Therefore, it is important to study the mechanisms of lead absorption and transportation for $\mathrm{Pb}$ resistance and lessening in tea plants.

To minimize the toxicity of heavy metals, plants have developed a complex network that controls metal uptake, transport, and storage [5]. Under heavy metal stress, some plants could accumulate metal ions in the roots and restrict the root-to-shoot transport to alleviate the toxicity in the overground part. Plant cells show defense against these heavy metals by chelating the toxic ions through a metal-chelating protein, as well as transporting into vacuoles or out the cell through metal membrane transporters [15].

The natural resistant-associated macrophage protein (NRAMP) is a kind of integral membrane transporter which usually has 10-12 putative transmembrane domains (TMDs), with consensus of residues between TMD-8 and TMD-9 [16]. The NRAMP was firstly discovered in mice [17] and has been identified as a divalent metal transporter in bacteria, fungi, insects, plants, and mammals $[18,19]$. The NRAMP protein family in Arabidopsis was classified into two subfamilies based on phylogenetic analysis. AtNRAMP1 and AtNRAMP6 form group I, while other AtNRAMP proteins belong to group II [20].

The NRAMP protein in plants is involved in the transport of multiple divalent cations, such as $\mathrm{Fe}^{2+}, \mathrm{Mn}^{2+}, \mathrm{Cu}^{2+}, \mathrm{Pb}^{2+}$, and $\mathrm{Cd}^{2+}[21,22]$. The roles of NRAMP proteins in plants have been analyzed in Arabidopsis thaliana [20], Oryza sativa [23], Populus alba [24], Phaseolus vulgaris [25], Theobroma cacao [26], and Brassica napus [27]. In Arabidopsis, AtNRAMP6 is an intracellular cadmium transporter [28], while AtNRAMP1 locates in the root plasma membrane and acts as a transporter for Mn. Additionally, AtNRAMP3 and AtNRAMP4 play a role in the transportation of Fe and Mn [29]. In rice, OsNRAMP3 and OsNRAMP5 have been identified as a transporter for $\mathrm{Mn}$ and Fe, respectively. OsNRAMP4 has been identified as the trivalent $\mathrm{Al}$ ion transporter [16]. Many papers have presented studies on the role of NRAMP proteins in Cd transport [16,23]. Overexpression of TtNRAMP1 could enhance the transport of $\mathrm{Cd}$ [30]. However, the role of the NRAMP protein family and the transport mechanisms for $\mathrm{Pb}$ in tea plants remain unknown.

In this study, we identified and characterized 11 NRAMP genes in the CSS reference genome database, and then the expression levels of 11 CsNRAMP genes were investigated in different tissues and under $\mathrm{Fe}, \mathrm{Mn}$, and $\mathrm{Pb}$ treatment. Also, we analyzed the subcellular location of CsNRAMP2 and CsNRAMP5. The characterization and expression analysis of the CsNRAMP family in tea plants could provide a theoretical basis for further studies of the response of the CsNRAMP family on $\mathrm{Pb}$ treatment in tea plants.

\section{Results}

\subsection{Identification and Characteristics of CsNRAMPs}

Thirteen candidate CsNRAMP full-length sequences containing the NRAMP domain were identified in a Blast search of the tea plant genome. However, TEA002429.1 and TEA017931.1 were excluded because they are homologous with ethylene-insensitive protein 2 (EIN2) [20]. Thus, we identified 11 CsNRAMP genes in this study (Table 1).

The number of amino acids of 11 CsNRAMP proteins ranged from 279 (CsNRAMP7) to 1373 (CsNRAMP11), while the molecular weight varied from 30,575.14-150,318.02 Da. The theoretical pIs of CsNRAMP proteins were various from 4.94-9.18. The exon numbers of CsNRAMPs ranged from 3-17. And the transmembrane domains of CsNRAMPs ranged from 3-12 (Table 1). 
Table 1. Bioinformatics analysis of CSNRAMP genes.

\begin{tabular}{|c|c|c|c|c|c|c|c|}
\hline Name & Gene ID & $\begin{array}{c}\text { Number of } \\
\text { Amino Acids }\end{array}$ & $\begin{array}{l}\text { Molecular } \\
\text { Weight }\end{array}$ & $\begin{array}{l}\text { Theoretical } \\
\text { pI }\end{array}$ & $\begin{array}{c}\text { Exon } \\
\text { Number }\end{array}$ & $\begin{array}{l}\text { Transmembrane } \\
\text { Number }\end{array}$ & Subcellular Localization \\
\hline CsNRAMP1 & TEA000600.1 & 525 & $57,407.13$ & 5.23 & 4 & 11 & $\begin{array}{l}\text { Membrane bound } \\
\text { Vacuolar }\end{array}$ \\
\hline CsNRAMP2 & TEA000624.1 & 497 & $54,044.99$ & 4.94 & 5 & 9 & $\begin{array}{c}\text { Membrane bound } \\
\text { Vacuolar }\end{array}$ \\
\hline CsNRAMP3 & TEA009385.1 & 589 & $64,295.88$ & 8.75 & 13 & 10 & Plasma membrane \\
\hline CsNRAMP4 & TEA002435.1 & 485 & $52,482.32$ & 8.81 & 12 & 11 & Plasma membrane \\
\hline CsNRAMP5 & TEA017264.1 & 512 & $56,070.19$ & 8.05 & 12 & 11 & Plasma membrane \\
\hline CsNRAMP6 & TEA012361.1 & 721 & $80,236.18$ & 8.55 & 10 & 11 & $\begin{array}{c}\text { Membrane bound } \\
\text { Vacuolar }\end{array}$ \\
\hline CsNRAMP7 & TEA012584.1 & 279 & $30,575.14$ & 9.18 & 3 & 6 & $\begin{array}{c}\text { Membrane bound } \\
\text { Vacuolar }\end{array}$ \\
\hline CsNRAMP8 & TEA022476.1 & 902 & $98,625.55$ & 9.01 & 17 & 14 & Plasma membrane \\
\hline CsNRAMP9 & TEA025235.1 & 527 & $58,851.23$ & 8.17 & 5 & 6 & $\begin{array}{l}\text { Membrane bound } \\
\text { Vacuolar }\end{array}$ \\
\hline CsNRAMP10 & TEA032256.1 & 375 & $42,411.81$ & 8.36 & 5 & 3 & $\begin{array}{c}\text { Membrane bound } \\
\text { Vacuolar }\end{array}$ \\
\hline CsNRAMP11 & TEA011223.1 & 1373 & $150,318.02$ & 5.81 & 10 & 12 & $\begin{array}{l}\text { Membrane bound } \\
\text { Vacuolar* }\end{array}$ \\
\hline
\end{tabular}

*: asterisks correspond to the predicted result of the first 1200 amino acids of CsNRAMP11.

\subsection{Phylogenetic Analysis and Duplication Analysis of CsNRAMPs}

To explore the phylogenetic association among NRAMP homologs in plant genomes, the sequences of 24 proteins from Arabidopsis thaliana, Oryza sativa, and Camellia sinensis were used to align a phylogenetic tree analysis. According to AtNRAMP proteins, the 11 CsNRAMP proteins were divided into two groups (Figure 1). CsNRAMP3, CsNRAMP4, CsNRAMP5, and CsNRAMP8 belonged to group 1, while other CsNRAMPs were included in group 2. Additionally, it was predicted that the CsNRAMPs of G1 (group 1) would locate on the plasma membrane, and the CsNRAMPs of G2 (group 2) on the membrane-bound vacuolar. The exon numbers of G1 CsNRAMPs varied from 12-13, and the AtNRAMP1 and AtNRAMP6 from the same group displayed 11 and 13 exons [25]. In another group, most members contained no more than five exons, which was similar to those for which the exon numbers were restricted to four [25] (Table 1).

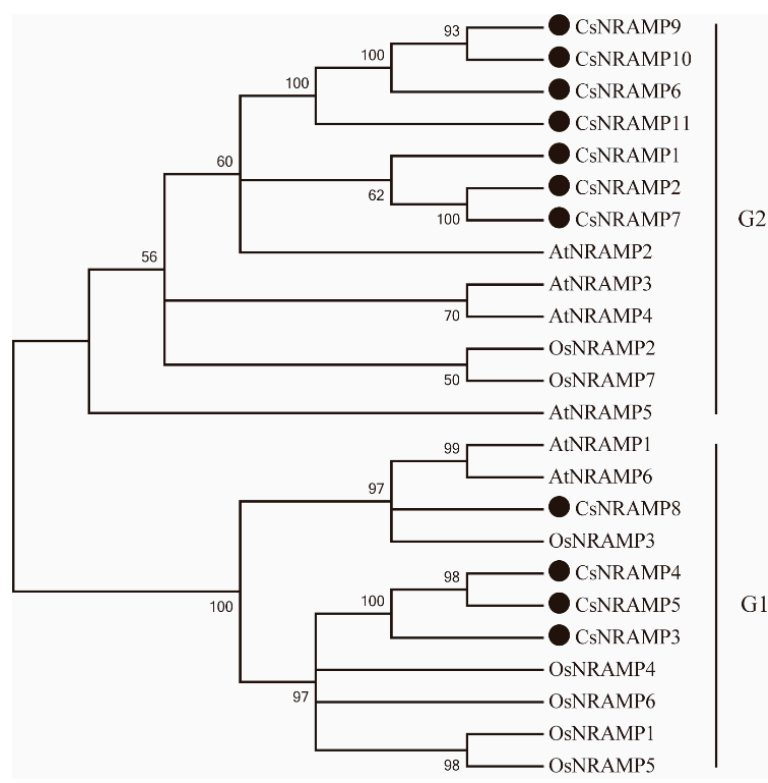

Figure 1. Unrooted phylogenetic tree analysis of NRAMP proteins from Camellia sinensis (Cs), Arabidopsis thaliana (At), and Oryza sativa (Os). Eleven sequences from Camellia sinensis are highlighted with a black circle. The two groups are indicated in the right panel. 
To explore the evolutionary patterns of the NRAMP gene family in the tea plant genome, gene duplication events were surveyed (Figure 2). Ten NRAMP genes were distributed unevenly in the 15 chromosomes in the tea plant genome. CsNRAMP7 was assembled to the scaffold. An analysis of tea plant NRAMP family genes revealed that three paralogous gene pairs (CsNRAMP2\&CsNRAMP11/CsNRAMP3\&CsNRAMP4/CsNRAMP9\&CsNRAMP10) existed in CSNRAMP family genes. (The ' $\&$ ' means the connector between duplicated gene pairs.)

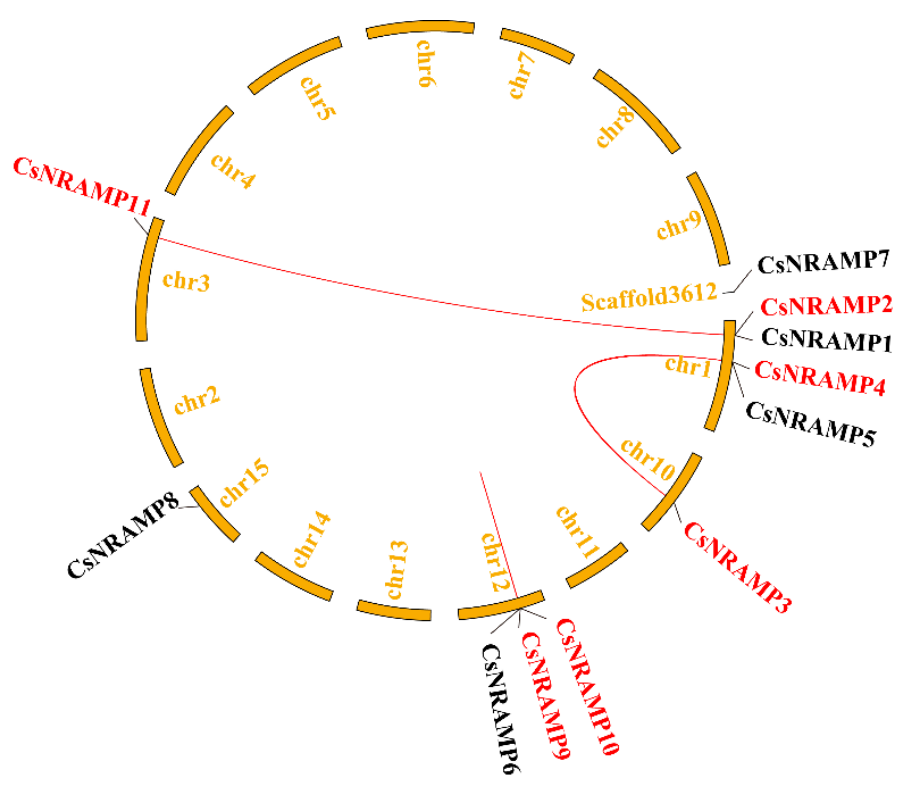

Figure 2. Duplication analysis of CsNRAMP genes. The red lines mean duplicated CsNRAMP gene pairs.

\subsection{Gene Structure and Conserved Motifs of CsNRAMP Proteins}

Previous studies on Arabidopsis and rice indicated that the NRAMP protein family usually contained 10-12 TMDs. Additionally, the most conserved amino acid residues, GQSSTITGGTYAGQXXMXGFLX, were located between the eighth and ninth transmembrane domains [31]. Most of the CsNRAMPs contained the conserved amino acid residues GQSSTxTGTYAGQFI MxGFLxLxxKKW, while CsNRAMP3 only had the latter part of residues, while CsNRAMP8 contained some other residues (Figure 3). Some NRAMP proteins contained broken NRAMP domains (Supplementary Materials, Figure S1).

There were 20 motifs identified in CsNRAMP proteins. The sequences of 20 motifs are shown in Supplementary materials, Table S1. All CsNRAMP proteins contained motif 1 and 4, while most of the CsNRAMP proteins in group 2 had motif 2, 3, 5, 6, 7, 8, and 14; and all of CsNRAMP proteins in group 1 had motif 2, 3, 5, 8, 10, 11, 15, 17, 19 and 20 (Figure 4). Only CsNRAMP9 and CsNRAMP10 proteins contained motif 12 and motif 13; motif 16 only existed in CsNRAMP6 and CsNRAMP9 proteins and motif 18 only existed in CsNRAMP1, CsNRAMP2 and CsNRAMP11 proteins. The conserved amino acid residues (Figure 3) consisted of motif 2 (GQFIMxGFLxLxxKKW) and motif 6 (GQSSTxTG) (Figure 5). All members of group 1 lacked the conserved motif 6 (GQSSTxTG). 

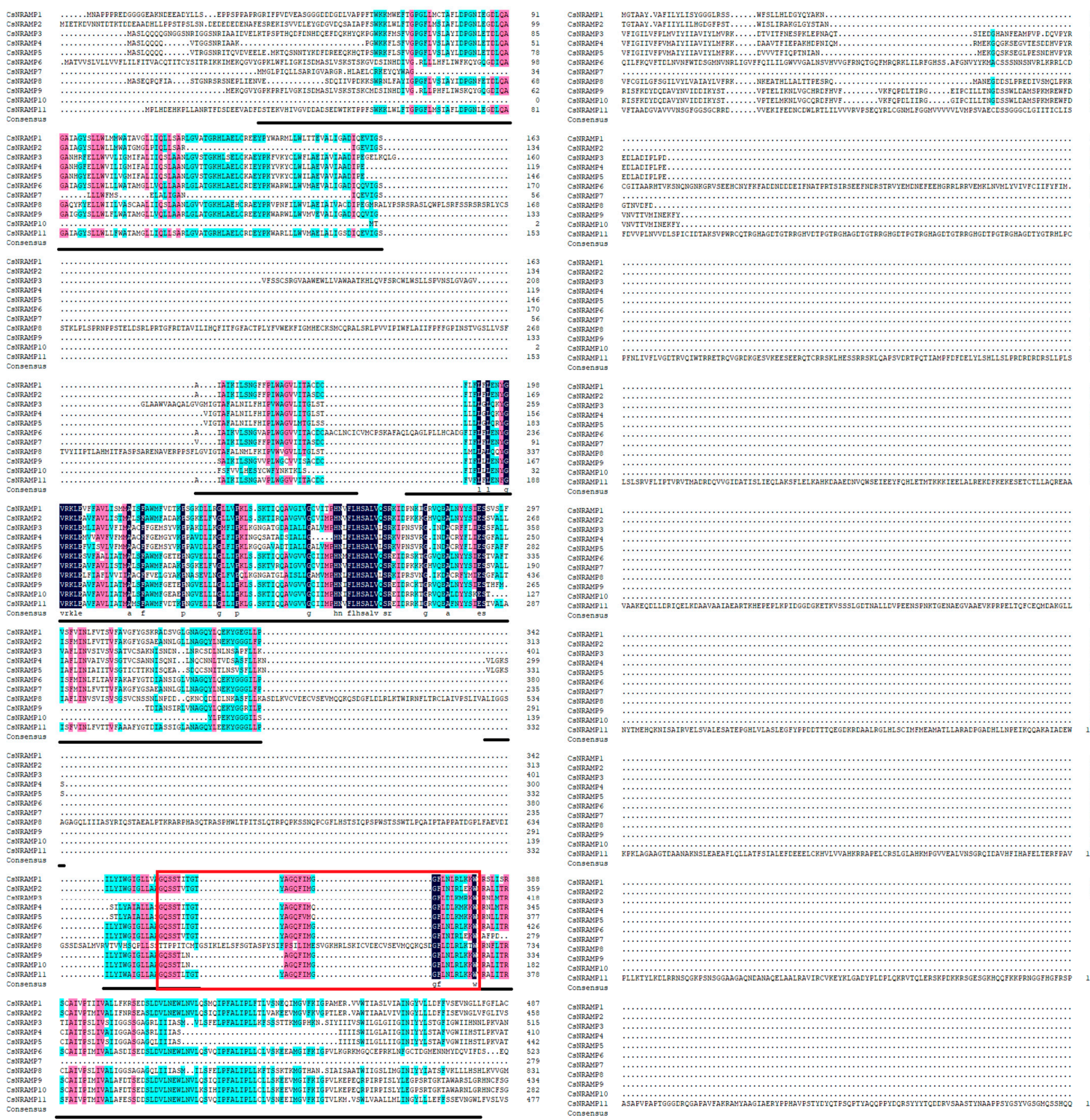

Figure 3. Multiple alignments of CsNRAMP proteins. The residues of NRAMP domain were underlined. And red rectangular frames mean the conserved residues. 


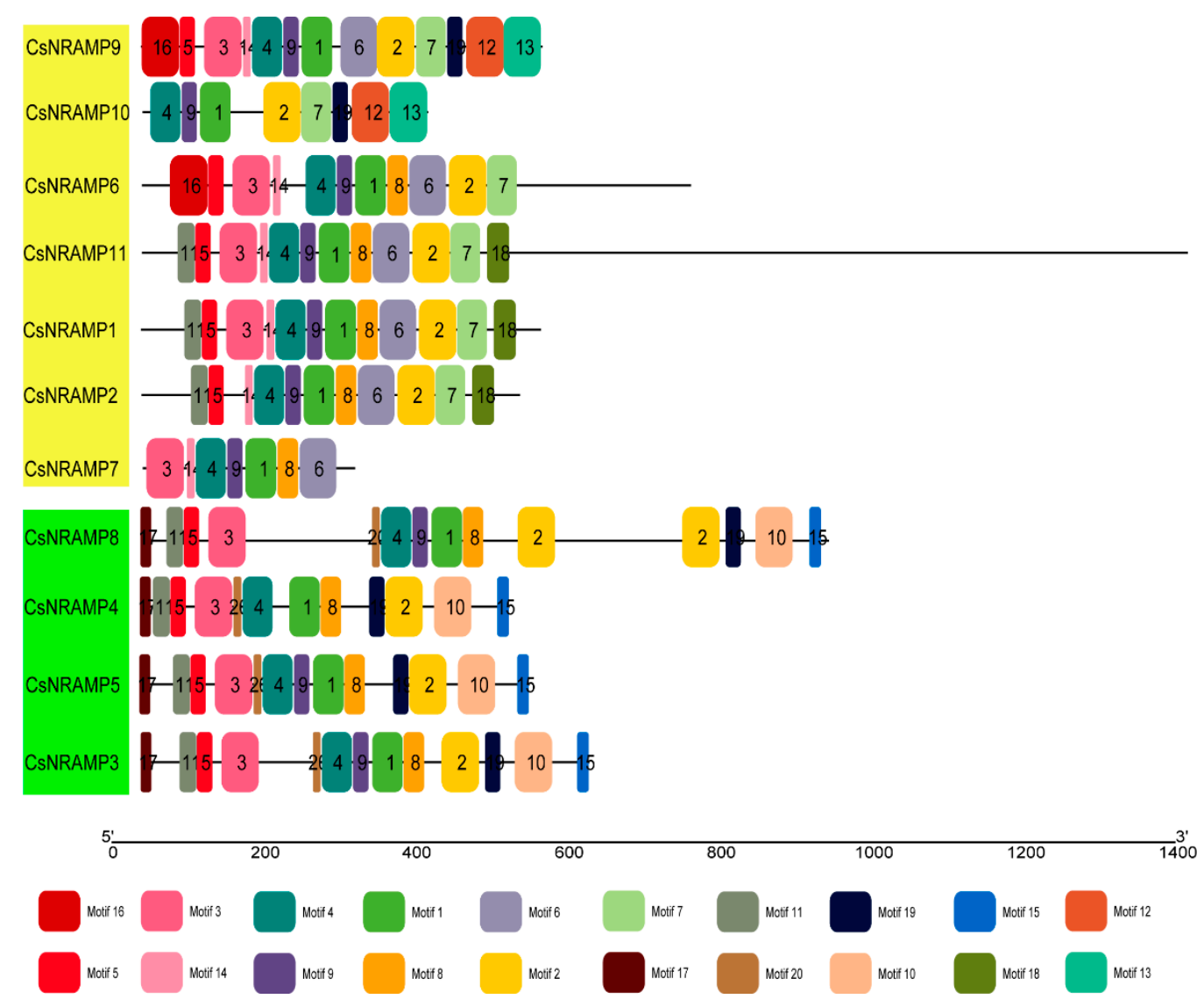

Figure 4. Motif analysis of CsNRAMP proteins. Boxes in different colors are used to distinguish different motif. CsNRAMP proteins were divided into two groups with yellow and green colors based on phylogenetic tree analysis.
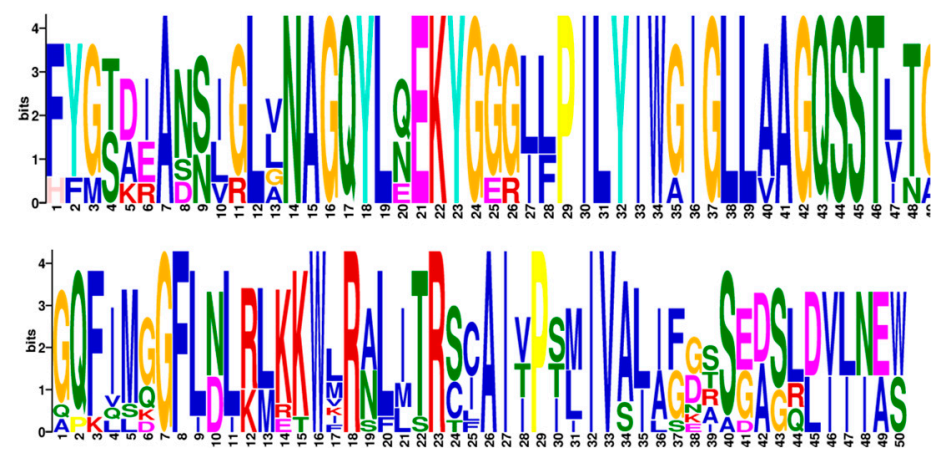

Figure 5. Sequence logos of conserved amino acid residues sequences in Camellia sinensis. The above one indicates motif 6 , the bottom one means motif 2. MEME motifs are displayed by stacks of letters at each site. The $x$-axis represents the width of the motif and the $y$-axis represents the bits of each letter.

\subsection{Expression Analysis of CsNRAMP Genes in Different Tissues}

We detected the expression of 11 CsNRAMP genes in different tissues of the tea plant. The results indicate that different genes exhibit different expression patterns (Figure 6). Three genes, CsNRAMP3, CsNRAMP4, and CSNRAMP5, showed extremely high specificity in the root, while four, CsNRAMP1, CsNRAMP2, CsNRAMP10, and CsNRAMP11, were highly expressed in the leaf, and CSNRAMP6 and CSNRAMP9 were highly expressed in the stem. Also, two genes, CsNRAMP7 and CsNRAMP8, exhibited high expression 
in both shoot and leaf. The genes in group 1, including CsNRAMP3, CsNRAMP4, and CSNRAMP5, showed similar expression patterns and reached higher expression levels in the root. Conversely, the genes belonging to group 2 were highly expressed in other tissues, such as stem and leaf. The CsNRAMP proteins might play different roles in the transportation of metals.
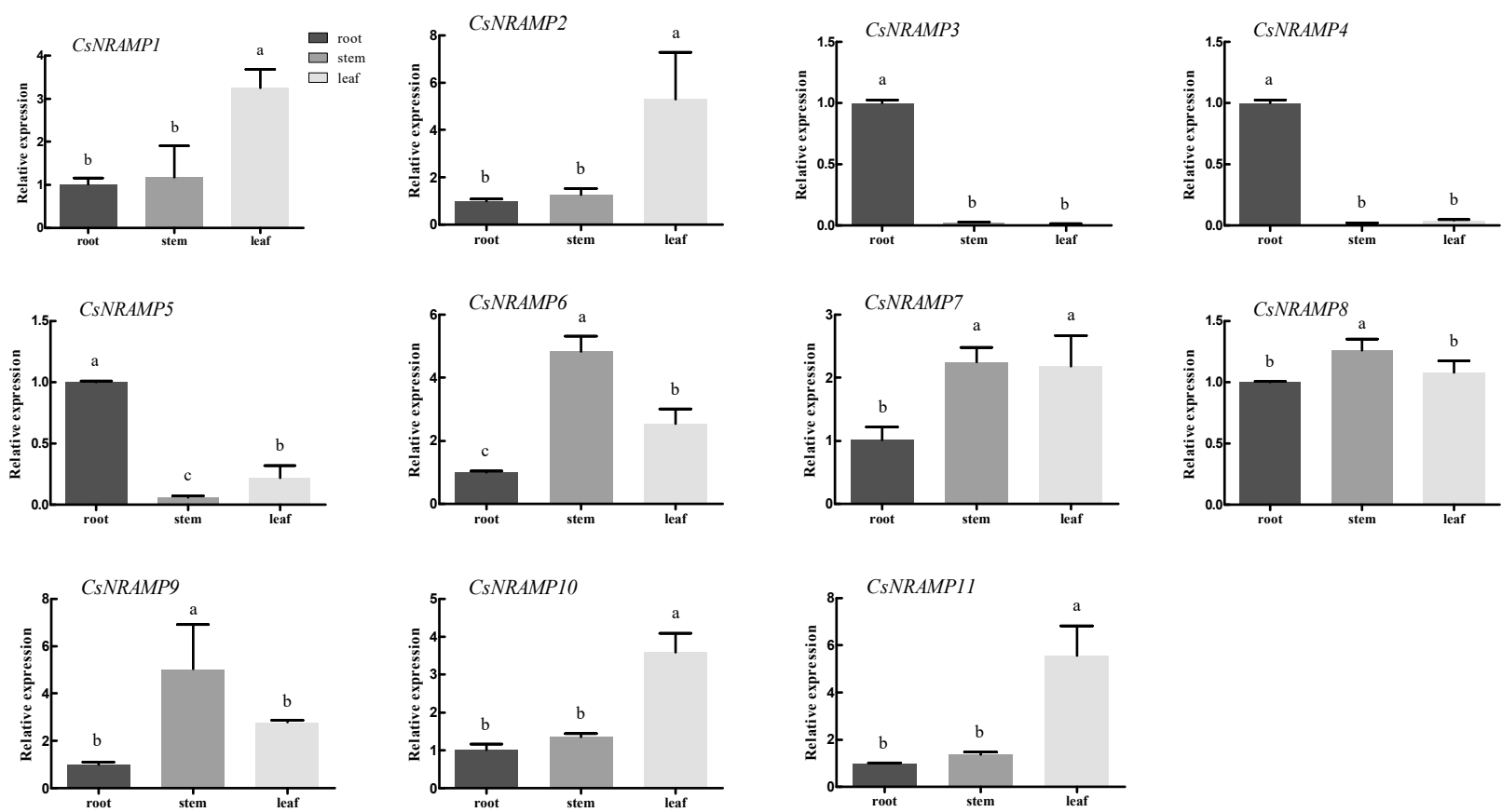

Figure 6. Expression of 11 CsNRAMP genes of Camellia sinensis in different tissues (root, stem, and leaf) of the tea plant. The expression level of tea actin was used as the internal control to standardize the RNA samples for each reaction. Error bars represent the mean values of three replicates \pm standard deviation (SD). Different lowercase letters indicate significant differences at $p<0.05$.

\subsection{Expression Analysis of CsNRAMP Genes under Lead Stress}

To investigate potential responses of CsNRAMPs to $\mathrm{Pb}$ treatment, expression patterns were detected by qRT-PCR in tea plants exposed to Pb. We noticed that CsNRAMP1, which prefers to express in leaves, showed an extremely low expression level in roots, while CsNRAMP3 CsNRAMP4 and CsNRAMP5 exhibited extremely low expression in leaves under $\mathrm{Pb}$ treatment.

In leaves, the expression of CsNRAMP2 was downregulated earlier and then upregulated at $50 \mathrm{mg} / \mathrm{L} \mathrm{Pb}$ treatment, and showed a double-peak pattern at $100 \mathrm{mg} / \mathrm{L} \mathrm{Pb}$ treatment. Additionally, CsNRAMP2 was significantly induced at $500 \mathrm{mg} / \mathrm{L} \mathrm{Pb}$ treatment for 1 and 7 days. The expression of CsNRAMP1 was downregulated under low Pb concentrations $(\leq 100 \mathrm{mg} / \mathrm{L})$, but increased at first and then decreased at high concentration. CsNRAMP10 showed a pattern that decreased and then increased at low Pb concentration, while it could be induced by high concentration. CsNRAMP11 expression, except at $100 \mathrm{mg} / \mathrm{L}$ for 1 day, was downregulated by $\mathrm{Pb}$ treatment. The expression of CsNRAMP7 and CsNRAMP9 exhibited a tendency that was downregulated and then increased. The expression of CsNRAMP6 and CsNRAMP8 was decreased (Figure 7A and Supplementary Materials, Figure S2). 


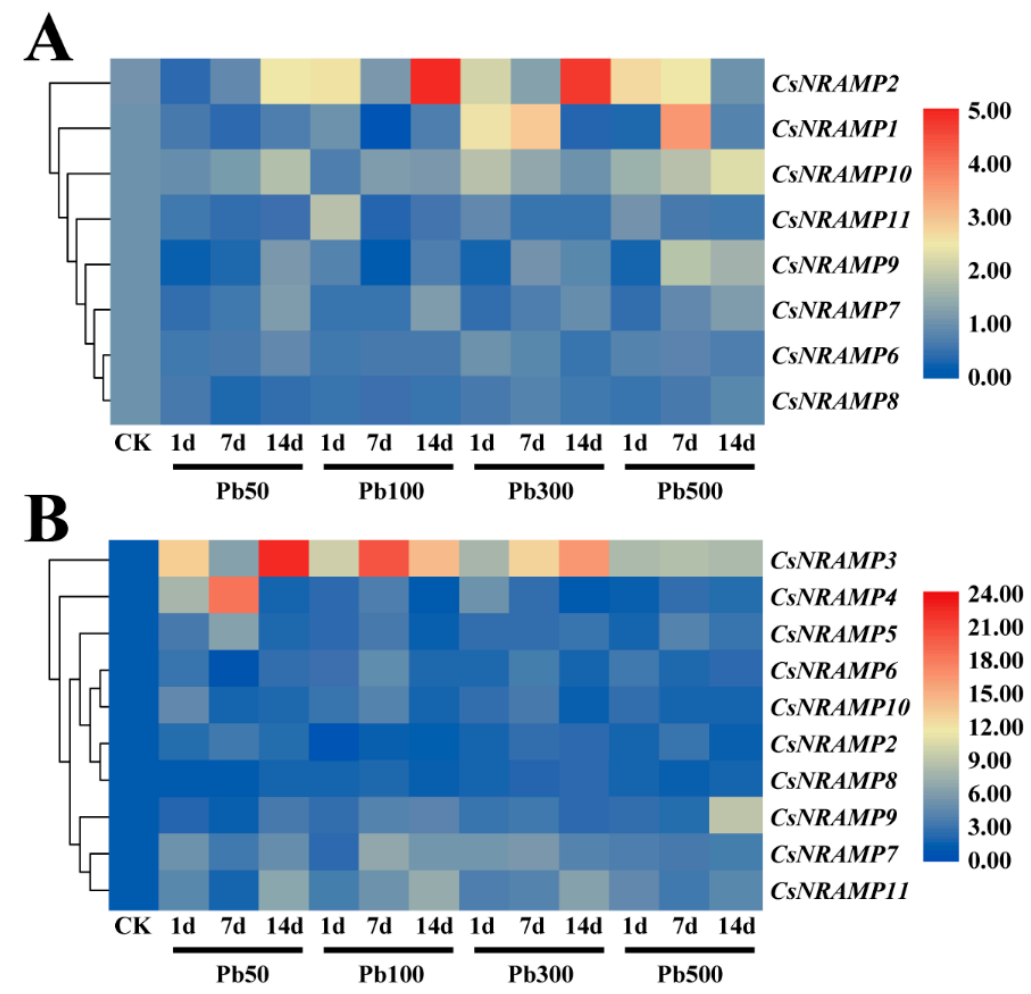

Figure 7. Expression of 11 CsNRAMP genes of Camellia sinensis under Pb treatment. (A) Expression heatmap of 8 CsNRAMP genes under different concentrations of $\mathrm{Pb}$ treatment in leaves. (B) Expression heatmap of 10 CsNRAMP genes under different concentrations of $\mathrm{Pb}$ treatment in roots. The expression level of tea actin was used as the internal control to standardize the RNA samples for each reaction and the expression at 0 day was set as CK. The underline with marks of Pb50, Pb100, Pb300, and $\mathrm{Pb} 500$ indicated that the concentration of $\mathrm{Pb}$ treatment was $50 \mathrm{mg} / \mathrm{L}, 100 \mathrm{mg} / \mathrm{L}, 300 \mathrm{mg} / \mathrm{L}$, and $500 \mathrm{mg} / \mathrm{L}$, respectively. And the sampling time after each treatment was 1 day, 7 days, and 14 days. Relative expression levels were shown in color as the scale.

In roots, CSNRAMP3 was significantly expressed in response to lead stress, and showed a double-peak pattern. At 50 and $100 \mathrm{mg} / \mathrm{L} \mathrm{Pb}$ treatment, the expression of CsNRAMP4 and CsNRAMP5 was upregulated and showed the highest transcriptional levels at 7 days, before decreasing. However, CSNRAMP4 and CSNRAMP5 were induced and stably expressed at $500 \mathrm{mg} / \mathrm{L}$ and $300 \mathrm{mg} / \mathrm{L} \mathrm{Pb}$ treatment. The CsNRAMP6 and CSNRAMP10 showed an initial increase, but subsequently decreased under $\mathrm{Pb}$ treatments. CsNRAMP2 and CsNRAMP8 showed a slight increase after treatment. The expression of CsNRAMP7, CsNRAMP9, and CsNRAMP11 increased, and CsNRAMP9 was highly expressed at $500 \mathrm{mg} / \mathrm{L}$ for 14 days. (Figure 7B and Supplementary Materials, Figure S3).

\subsection{Cloning of CsNRAMP2 and CsNRAMP5 and Subcellular Localization}

To understand the subcellular location of the CsNRAMP2 and CsNRAMP5 proteins, full-length CsNRAMP2 and CsNRAMP5 without ending codes were cloned and inserted into EGFP-fusion expression vector. The recombinant plasmid with a plasma membrane (PM) marker AtPIP2A-mCherry was cotransformed through transient infiltration to tobacco epidermis cells. The results indicated that both EGFP-CsNRAMP2 and EGFP-CsNRAMP5 fusion proteins were located in the plasma membrane (Figure 8). The subcellular localization of EGFP-CsNRAMP5 was coincident with the result predicted on the SoftBerry ProtComp website. However, the subcellular localization of CsNRAMP2 was the plasma membrane, which was different from the prediction of the SoftBerry ProtComp website. 


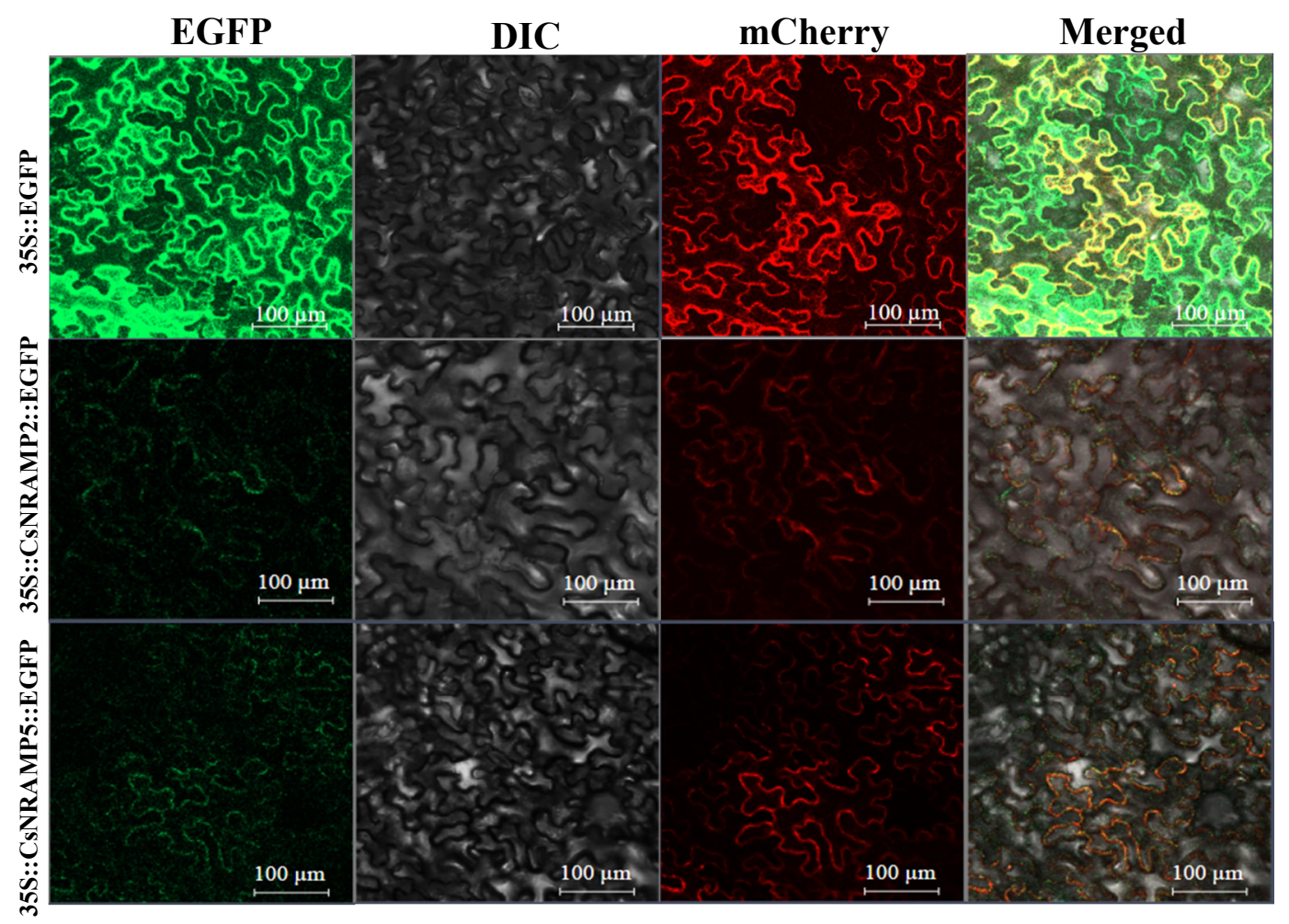

Figure 8. Subcellular location of the fusion protein 35S::CsNRAMP2::EGFP and 35S::CsNRAMP2::EGFP in tobacco epidermis cells (through transient infiltration). The vector 35S::EGFP was used as the control, and the AtPIP2A-mCherry was used as a plasma membrane (PM) marker. Bar $=100 \mu \mathrm{m}$.

\section{Discussion}

Based on the conserved domain of AtNRAMPs (PF01566), we identified 11 NRAMP genes from the Tea Plant Genome Database. Since the NRAMP genes were divided into two groups in Arabidopsis, we also split the CsNRAMPs into two groups [20]: CsNRAMP3, CsNRAMP4, CsNRAMP5, and CsNRAMP8 in group 1, and other CsNRAMPs in group 2.

The NRAMP proteins have been reported to contain 10-12 transmembrane domains and consist of around 500 amino acid residues in different species. For example, OsNRAMP proteins contained 518-550 amino acid residues and 10-12 transmembrane regions in rice [16]. Similarly, it was verified that there are 12 transmembrane domains in PvNRAMPs, and that the length of amino acid residues ranged from 507-554 [25]. However, in this study, we learned that CsNRAMP proteins contain 3-12 transmembrane regions and consist of 279-1373 amino acid residues. This may have been due to a broken NRAMP domain (Supplementary materials, Figure S1) or variation among species. This result was similar to those of several members of BnNRAMPs, which only carried 100-200 amino acid residues [29]. NRAMP proteins have been reported to carry consensus residues between TMD8 and TMD9, e.g., the AtNRAMP proteins in Arabidopsis contained GQSSTITGTY AGQXXMXGFLX, while PvNRAMP proteins in Phaseolus vulgaris carried GQSSTITGTYAGQFIMGGFLN $[25,28]$. According to our results, the CsNRAMP proteins carried similar consensus residues, i.e., GQSSTxTGTYAGQFIMxGFLxLxxKKW, which consisted of motif 2 (GQFIMxGFLxLxxKKW) and 6 (GQSSTxTG). For motif analysis, motifs 6 and 14 are only present in G2 CsNRAMP proteins, while motifs 10, 15, 17, and 20 are only present in the G1 CsNRAMP proteins; this may be related to the differences between groups. We also analyzed motifs of the tea plant, Arabidopsis and rice, finding that motifs 17 and 18 discriminated groups 1 and 2 (Supplementary Materials, Figure S4). This was similar to the results of NRAMPs of cacao, Arabidopsis, and rice, which were split into three clusters with a comparison of conserved motifs [26]. 
In this study, we learned that G1 genes, CsNRAMP3, CsNRAMP4, and CsNRAMP5, were extraordinarily expressed in roots, while G2 genes showed higher expression levels in the stems and leaves. The NRAMP family genes of plants have been proven to function on a wide range of divalent metal ions, including absorption, transportation, and homeostasis [32]. For example, AtNRAMP6 functions in the lateral root and young leaves of Arabidopsis [33]. AtNRAMP1 plays a pivotal role in the Fe transportation in roots [34]. SaNRAMP1 can be strongly expressed in the young shoots and transportation of $\mathrm{Cd}, \mathrm{Mn}$, and $\mathrm{Zn}$ [35]. To sum up, the members of G1 CsNRAMPs were mainly expressed in the root, combing with their plasma membrane localization (Table 1), indicated that G1 CsNRAMPs may participate in the absorption process of metal ions. AtNRAMP3 and AtNRAMP4 are located on vacuolar membranes and function in vacuolar Fe mobilization [36,37], while it was predicted that the G2 members would be located on the membrane bound vacuolar, which might be related to the transport and distribution of metal in the tea plant.

In plants, NRAMPs were found to participate in multiple divalent metal transportation [22]. The expression of BnNRAMP2;1 and BnNRAMP4;2 could be increased after exposure to $\mathrm{Cd}$ [38]. Nrat1 characterized as Al transporters in rice could be highly expressed in response to Al stress [39]. LeNRAMP1 could be significantly induced by iron deficiency [40]. AtNRAMP3 and AtNRAMP4 proteins have been proved to function in the transportation of Fe and Mn [36,37]. OsNRAMP6 and OsNRAMP5 have been shown to be involved in the uptake of Fe and Mn [16]. According to our results, the members of CsNRAMPs could be differently upregulated or downregulated under $\mathrm{Pb}$ treatment. The expression levels of most CsNRAMPs in leaves were lower than in roots. This may have been due to the fact that plants restrict most of the heavy metals to the roots to alleviate toxicity on shoots and leaves [15]. In leaves, the expression levels of CsNRAMP1, CsNRAMP2, CsNRAMP9, and CSNRAMP10 were upregulated, and CsNRAMP2 showed extremely sensitive response to $\mathrm{Pb}$ treatment. In roots, the transcription levels of CsNRAMP3, CsNRAMP4, CsNRAMP5, CsNRAMP7, and CsNRAMP9 were accumulated after exposure to $\mathrm{Pb}$. In particular, the expression of CsNRAMP3 was sharply increased under Pb treatment. This indicates that these genes may play a role in $\mathrm{Pb}$ transportation. Interestingly, some CsNRAMPs showed a double-peak pattern after Pb treatment, which may be have been due to a complicated interaction mechanism in the tea plants. The MTs also showed a similar expression pattern under Cd stress [41].

Regarding G2 members, the NRAMP domain of CsNRAMP3 protein was broken (Supplementary Materials, Figure S1). CsNRAMP7, CsNRAMP9, and CsNRAMP10 only contained six, six, and three transmembrane regions respectively, in contrast to 10-12 TMDs. Furthermore, CsNRAMP1 and CsNRAMP2 were close on the phylogenetic tree, and the expression level of CSNRAMP2 increased more than CSNRAMP1 under Pb treatment in leaves. Regarding G1 members, both expression levels CsNRAMP4 and CsNRAMP5 were increased by $\mathrm{Pb}$ treatment in roots; however, the expression of CSNRAMP5 under $100 \mathrm{mg} / \mathrm{L}$ $\mathrm{Pb}$ treatment for 14 days was increased around 12 fold in leaves (Supplementary Materials, Figure S2). Overall, CsNRAMP2 from G2 and CsNRAMP5 from G1 were chosen for further studies, since NRAMP genes function in metal ion transportation, especially Fe [42]. In this study, the responses of CsNRAMP2 and CsNRAMP5 to Fe and Mn treatments were studied. The results showed that Fe treatment (400 $\mu \mathrm{M}$ EDTA-Fe) increased the expression of CsNRAMP2 and decreased the expression of CsNRAMP5, while Mn treatment up-regulated the expression of CSNRAMP5 in roots (Supplementary Materials, Figure S5). As shown in Figure 1, the CSNRAMP2 protein clustered together into a small phylogenetic branch with AtNRAMP2, AtNRAMP3, and AtNRAMP4, which are known to be implicated in the transportation of Fe and Mn [36,37]. Thus, CsNRAMP2 may play a role in transporting Fe and $\mathrm{Pb}$. Meanwhile, CsNRAMP5 was close to OsNRAMP6 and OsNRAMP5, which have been shown to be involved in the uptake of Fe and Mn [16]. CsNRAMP5 may participate in the transportation of $\mathrm{Mn}$ and $\mathrm{Pb}$. All of these speculations need further study.

Proteins of the NRAMP family located on different organelles show various functions. For example, AtNRAMP6 has been shown to be located in the Golgi/trans-Golgi network 
and play an important role in intracellular Fe homeostasis [33]. Additionally, AtNRAMP3 and AtNRAMP4 are located on vacuolar membranes and contribute to Fe mobilization [37]. OsNRAMP1 on the plasma membrane of endodermis and pericyle cells may assist in the loading of arsenic from roots to shoots mobilization [32]. In this study, we analyzed the subcellular locations of the CSNRAMP2 and CsNRAMP5 proteins. The results indicated that both CSNRAMP2 and CSNRAMP5 fusion proteins are located in the plasma membrane. CsNRAMP2 and CsNRAMP5 fusion proteins may function in the transmembrane transport of metal ions.

In summary, we first identified 11 NRAMP genes in a genome-wide survey of the tea plant. The expression profiles of CSNRAMP genes varied in tea plant tissues, implying that CSNRAMPs perform functions in specific tissues. Based on the expression analysis under $\mathrm{Pb}$ treatment, we speculated that certain CsNRAMP genes might contribute to $\mathrm{Pb}$ transportation. Furthermore, the subcellular localization analysis in tobacco epidermis cells confirmed the plasma membrane localization of CsNRAMP2 and CsNRAMP5 proteins. These results provide basic information for understanding the functions of the NRAMP family in tea plants, and suggest potential future study directions regarding transport signaling pathways.

\section{Materials and Methods}

\subsection{Identification of CsNRAMP Family Genes in the Tea Plant}

The sequences of NRAMP genes of the tea plant were obtained derived from the Tea plant Genome Database (http:/ / tpia.teaplant.org/download.html (accessed on 1 October 2019)). The NRAMP domain (PF01566) of six AtNRAMP proteins was used to search the local tea protein database for target sequences by using Bioedit version 7.0.9 software. Pfam Database (http:/ / pfam.xfam.org/ (accessed on 30 October 2019)) and SMART (http:/ / smart.embl-heidelberg.de/ (accessed on 30 October 2019)) were used to determine predicted protein as a member of the transporter gene family.

\subsection{Characterization of CSNRAMP Proteins}

Six Arabidopsis NRAMP protein sequences and seven rice NRAMP protein sequences were downloaded from the Arabidopsis Information Resource (TAIR) (https://www. arabidopsis.org/ (accessed on 23 December 2020)) and The Rice Annotation Project Database (https:/ / rapdb.dna.affrc.go.jp/download/irgsp1.html (accessed on 23 December 2020)). MEGA version 7.0 was used to construct a bootstrap neighbor-joining(NJ) phylogenetic tree for NRAMP protein of Arabidopsis, rice, and tea with MUSCLE alignment and 1000 bootstrap replicates [43]. The duplication events were analyzed using the DupGen_finder [44], and then visualized using TBtools v.1.0692 [45]. The physicochemical properties of the CsNRAMP proteins were analyzed using ExPASy-ProtParam (https: / / web.expasy.org / protparam/ (accessed on 22 July 2020)). The exon organization was determined using by TBtools v.1.0692 [45]. Prediction of subcellular localization and transmembrane helices of the proteins encoded by CSNRAMP genes were displayed by SoftBerry ProtComp (http: / / linux1.softberry.com/berry.phtml?topic=protcomppl\&group=programs\& subgroup $=$ proloc (accessed on 22 July 2020)) and TMHM M Server v.2.0 (http:/ / www. cbs.dtu.dk/services/TMHMM-2.0/ (accessed on 22 July 2020)). The conserved motifs and domains of CsNRAMP proteins were obtained using the MEME $\left(\mathrm{E}<1 \mathrm{e}^{-10}\right)$ online tool (http:/ / meme-suite.org/tools/meme (accessed on 5 January 2021)) and Pfam (http:/ / pfam.xfam.org/ (accessed on 5 January 2021)) respectively, and then visualized by visualizing the domain pattern of TBtools v.1.0692 [45]. Finally, multiple sequence alignments of the CsNRAMP proteins were performed using DNAMAN version 7.0.

\subsection{Plant Materials and Treatments}

One-year-old seedlings of tea plants "zhongcha108" were grown in a growth chamber at the Tea Science Research Institute of Nanjing Agricultural University (Nanjing, China). These seedlings were cultivated in a nutrient solution [46], and were precultured in $1 / 4$ 
nutrient solution and then $1 / 2$ nutrient solution for acclimatization. Then, seedlings were cultured in a total nutrient solution for about two weeks. After that, the leaves, stems, and roots were harvested for expression analysis of CsNRAMP genes of the tea plant.

Regarding lead treatment, the seedlings were cultivated in a nutrient solution containing 50,100,300, $500 \mathrm{mg} / \mathrm{L} \mathrm{Pb}^{2+}\left(\mathrm{Pb}\left(\mathrm{NO}_{3}\right)_{2}\right)$, and then the leaves and roots were collected at 1,7 , and 14 days after treatment. The CK leaves and roots were collected before lead treatment.

To investigate the possible function of CsNRAMP2 and CsNRAMP5 in response to divalent metal, tea plant seedlings were exposed to the treatments of iron and manganese for 7 days, respectively. For iron treatment, excess Fe $(200 \mu \mathrm{M}$ or $400 \mu \mathrm{M}$ EDTA-Fe $)$ was added for iron treatment. Additionally, $200 \mu \mathrm{M}$ or $400 \mu \mathrm{M} \mathrm{MnSO} 4 \cdot \mathrm{H}_{2} \mathrm{O}$ was added for $\mathrm{Mn}$ treatment. The seedlings cultivated in full-strength nutrient solution were sampled as the control (CK, $6.27 \mu \mathrm{M}$ Fe-Na-EDTA and $\left.18.22 \mu \mathrm{M} \mathrm{MnSO}_{4} \cdot \mathrm{H}_{2} \mathrm{O}\right)$.

All samples were frozen in liquid nitrogen, then stored at $-80{ }^{\circ} \mathrm{C}$ for the following experiments. All experiments were repeated with three biological and technical replicates.

\subsection{Expression Profile Analyses}

Total RNA was extracted from the leaves and roots using an RNA quick isolation kit (Aidlab, Beijing, China), and reverse transcription was performed with TransScript ${ }^{\circledR}$ One-Step gDNA Removal and cDNA Synthesis SuperMix (TransGen, Beijing, China). The expression of CsNRAMPs was analyzed by quantitative real-time PCR using the SYBR Premix Ex Taq II kit (Takara, Kusatsu, Japan). The primer pairs used for qRT-PCR were designed by Primer Premier 5.0 and $\beta$-actin was used as an internal control (Table S2). The qRT-PCR program was as follows: $95^{\circ} \mathrm{C}$ for $30 \mathrm{~s}, 40$ cycles at $95^{\circ} \mathrm{C}$ for $5 \mathrm{~s}, 60^{\circ} \mathrm{C}$ for $30 \mathrm{~s}$ [47]. All experiments were repeated with three biological and technical replicates. Relative gene expressions were calculated using the $2^{-\Delta \Delta C t}$ method [48].

\subsection{Subcellular Location Confirmation of CsNRAMP2 and CsNRAMP5}

The full open-reading frame sequences of CSNRAMP2 and CSNRAMP5 gene without the stop codon were amplified and then inserted into EGFP-fusion expression vector pCAMBIA 2300 at $\mathrm{XbaI}$ and SmaI site using Trelief ${ }^{\mathrm{TM}}$ SoSoo Cloning Kit Ver.2 (Tsingke, Beijing, China). The recombinant plasmid, EGFP-CSNRAMP2 or EGFP-CSNRAMP5, and empty vector were transformed into Agrobacterium tumefaciens strain GV3101 cells. Furthermore, we cotransformed GV3101 containing recombinant plasmids or empty vectors with a plasma membrane (PM) marker AtPIP2A-mCherry through transient infiltration to tobacco epidermis cells [49]. Plants were incubated in the dark overnight and normal cycle for two days and then detected using An LSM800 Ultra high-resolution confocal microscopy imaging system (Zeiss Co., Oberkochen, Germany) [50].

\subsection{Statistical Analysis}

Excel 2010, SPSS 20.0, and GraphPad Prism 5 were used to analyze the experimental data. Duncan's method was used for multiple comparisons of variance analysis, and $p<0.05$ indicated a significant difference.

Supplementary Materials: The following are available online at https:/ /www.mdpi.com/article/10 .3390 / plants10061055/s1, Figure S1: Domain analysis of CsNRAMP proteins. Figure S2: Expression of CsNRAMP2, -4 and -5 of Camellia sinensis in leaves. Figure S3: Expression of CsNRAMP1 of Camellia sinensis in roots. Figure S4 Motif analysis of NRAMP proteins from Camellia sinensis (Cs), Arabidopsis thaliana (At), and Oryza sativa (Os). Figure S5 Expression of CsNRAMP2 and CsNRAMP5 of Camellia sinensis (leaf and root) in response to Fe and Mn treatments. Table S1: Motif consensus. Table S2: Primers for expression analysis.

Author Contributions: Conceptualization, W.F. and X.Z.; methodology, Y.M. and F.L.; software, J.L.; validation, Y.D. and Z.H.; formal analysis, Z.H. and K.Z.; investigation, J.L.; re-sources, J.L.; data curation, J.L. and X.S.; writing —original draft preparation, J.L.; writing-review and editing, Z.Z. 
and X.Z.; visu-alization, F.L.; supervision, Y.M.; project administration, X.Z.; funding acquisition, W.F. and X.Z. All authors have read and agreed to the published version of the manuscript.

Funding: This research was funded by the National Key R \& D Program of China (2020YFE0202900), The National Natural Science Foundation of China (31870680, 31972460), the earmarked fund for China Agriculture Research System (CARS-19), the Key Research and Development Program of Jiangsu Province (BE2019379), Jiangsu Agriculture Science and Technology Innovation Fund (CX(20)2004), Innovation and Extension Projects of Forestry Science and Technology in Jiangsu Province (LYKJ-Changzhou(2020)03), Changzhou Science and Technology Support Program (Agriculture CE20202003), and Chuzhou Science and Technology Support Program (2020ZN009).

Institutional Review Board Statement: Not applicable.

Informed Consent Statement: Not applicable.

Data Availability Statement: Not applicable.

Acknowledgments: We would like to thank the Central Lab in College of Horticulture for providing the LSM800 confocal microscopy imaging system and Bio-Rad IQ5 Real Time PCR System.

Conflicts of Interest: The authors declare that they have no conflict of interest.

\section{References}

1. Han, W.-Y.; Shi, Y.-Z.; Ma, L.-F.; Ruan, J.-Y.; Zhao, F.-J. Effect of liming and seasonal variation on lead concentration of tea plant (Camellia sinensis (L.) O. Kuntze). Chemosphere 2007, 66, 84-90. [CrossRef] [PubMed]

2. Dang, M.V. Soil-plant nutrient balance of tea crops in the northern mountainous region, Vietnam. Agric. Ecosyst. Environ. 2005, 105, 413-418. [CrossRef]

3. Alkhatib, R.; Mheidat, M.; Abdo, N.; Tadros, M.; Al-Eitan, L.; Al-Hadid, K. Effect of lead on the physiological, biochemical and ultrastructural properties ofLeucaena leucocephala. Plant Biol. 2019, 21, 1132-1139. [CrossRef] [PubMed]

4. Mao, X.F. EFFECTS OF HEAVY METAL PB AND CD STRESS ON PHYSIOLOGICAL CHARACTERISTICS OF JAPANESE HONEYSUCKLE. Appl. Ecol. Environ. Res. 2019, 17, 6415-6427. [CrossRef]

5. Jalmi, S.K.; Bhagat, P.K.; Verma, D.; Noryang, S.; Tayyeba, S.; Singh, K.; Sharma, D.; Sinha, A.K. Traversing the Links between Heavy Metal Stress and Plant Signaling. Front. Plant Sci. 2018, 9, 12. [CrossRef]

6. Yadav, S.K. Heavy metals toxicity in plants: An overview on the role of glutathione and phytochelatins in heavy metal stress tolerance of plants. South Afr. J. Bot. 2010, 76, 167-179. [CrossRef]

7. Lal, N. Molecular Mechanisms and Genetic Basis of Heavy Metal Toxicity and Tolerance in Plants. In Plant Adaptation and Phytoremediation; Springer: Berlin/Heidelberg, Germany, 2010; pp. 35-58. [CrossRef]

8. Rucińska-Sobkowiak, R. Water relations in plants subjected to heavy metal stresses. Acta Physiol. Plant. 2016, 38, 257. [CrossRef]

9. Zazouli, M.A.; Bandpei, A.M.; Maleki, A.; Saberian, M.; Izanloo, H. Determination of Cadmium and Lead Contents in Black Tea and Tea Liquor from Iran. Asian J. Chem. 2010, 22, 1387-1393.

10. Wen, B.; Li, L.; Duan, Y.; Zhang, Y.; Shen, J.; Xia, M.; Wang, Y.; Fang, W.; Zhu, X. Zn, Ni, Mn, Cr, Pb and Cu in soil-tea ecosystem: The concentrations, spatial relationship and potential control. Chemosphere 2018, 204, 92-100. [CrossRef]

11. Schwalfenberg, G.; Genuis, S.J.; Rodushkin, I. The Benefits and Risks of Consuming Brewed Tea: Beware of Toxic Element Contamination. J. Toxicol. 2013, 2013, 1-8. [CrossRef]

12. Jin, C.W.; He, Y.F.; Zhang, K.; Di Zhou, G.; Shi, J.L.; Zheng, S.J. Lead contamination in tea leaves and non-edaphic factors affecting it. Chemosphere 2005, 61, 726-732. [CrossRef] [PubMed]

13. Lv, Y.M.; Yang, J.P.; He, J.; Zhao, X.; Ye, X.Y. Effects of Phosphate Fertilizers on Bioavailable Lead in Tea Garden Soil and Lead Absorption and Accumulation by Tea Plants. Appl. Mech. Mater. 2014, 651-653, 231-235. [CrossRef]

14. Chen, Y.; Xu, J.; Yu, M.; Chen, X.; Shi, J. Lead contamination in different varieties of tea plant (Camellia sinensis L.) and factors affecting lead bioavailability. J. Sci. Food Agric. 2010, 90, 1501-1507. [CrossRef] [PubMed]

15. DalCorso, G.; Fasani, E.; Manara, A.; Visioli, G.; Furini, A. Heavy Metal Pollutions: State of the Art and Innovation in Phytoremediation. Int. J. Mol. Sci. 2019, 20, 3412. [CrossRef]

16. Mani, A.; Sankaranarayanan, K. In Silico Analysis of Natural Resistance-Associated Macrophage Protein (NRAMP) Family of Transporters in Rice. Protein J. 2018, 37, 237-247. [CrossRef]

17. Vidal, S.M.; Malo, D.; Vogan, K.; Skamene, E.; Gros, P. Natural resistance to infection with intracellular parasites: Isolation of a candidate for Bcg. Cell 1993, 73, 469-485. [CrossRef]

18. Pinner, E.; Gruenheid, S.; Raymond, M.; Gros, P. Functional Complementation of the Yeast Divalent Cation Transporter Family SMF by NRAMP2, a Member of the Mammalian Natural Resistance-associated Macrophage Protein Family. J. Biol. Chem. 1997, 272, 28933-28938. [CrossRef]

19. Gunshin, H.; MacKenzie, B.; Berger, U.V.; Gunshin, Y.; Romero, M.F.; Boron, W.F.; Nussberger, S.; Gollan, J.L.; Hediger, M.A. Cloning and characterization of a mammalian proton-coupled metal-ion transporter. Nat. Cell Biol. 1997, 388, 482-488. [CrossRef] 
20. Mäser, P.; Thomine, S.; Schroeder, J.I.; Ward, J.M.; Hirschi, K.; Sze, H.; Talke, I.N.; Amtmann, A.; Maathuis, F.J.; Sanders, D.; et al. Phylogenetic Relationships within Cation Transporter Families of Arabidopsis. Plant Physiol. 2001, 126, 1646-1667. [CrossRef]

21. Vatansever, R.; Filiz, E.; Ozyigit, I.I. In silico analysis of Mn transporters (NRAMP1) in various plant species. Mol. Biol. Rep. 2016, 43, 151-163. [CrossRef]

22. Bressler, J.P.; Olivi, L.; Cheong, J.H.; Kim, Y.; Bannona, D. Divalent Metal Transporter 1 in Lead and Cadmium Transport. Ann. N. Y. Acad. Sci. 2004, 1012, 142-152. [CrossRef] [PubMed]

23. Chang, J.; Huang, S.; Yamaji, N.; Zhang, W.; Ma, J.F.; Zhao, F. OsNRAMP1 transporter contributes to cadmium and manganese uptake in rice. Plant, Cell Environ. 2020, 43, 2476-2491. [CrossRef] [PubMed]

24. Chen, H.-M.; Wang, Y.-M.; Yang, H.-L.; Zeng, Q.-Y.; Liu, Y.-J. NRAMP1 promotes iron uptake at the late stage of iron deficiency in poplars. Tree Physiol. 2019, 39, 1235-1250. [CrossRef]

25. Ishida, J.K.; Caldas, D.G.; Oliveira, L.R.; Frederici, G.C.; Leite, L.M.P.; Mui, T.S. Genome-wide characterization of the NRAMP gene family in Phaseolus vulgaris provides insights into functional implications during common bean development. Genet. Mol. Biol. 2018, 41, 820-833. [CrossRef] [PubMed]

26. Ullah, I.; Wang, Y.; Eide, D.J.; Dunwell, J.M. Evolution, and functional analysis of Natural Resistance-Associated Macrophage Proteins (NRAMPs) from Theobroma cacao and their role in cadmium accumulation. Sci. Rep. 2018, 8, 1-15. [CrossRef]

27. Zhang, X.D.; Meng, J.G.; Zhao, K.X.; Chen, X.; Yang, Z.M. Annotation and characterization of Cd-responsive metal transporter genes in rapeseed (Brassica napus). BioMetals 2017, 31, 107-121. [CrossRef]

28. Cailliatte, R.; Lapeyre, B.; Briat, J.; Mari, S.; Curie, C. The NRAMP6 metal transporter contributes to cadmium toxicity. Biochem. J. 2009, 422, 217-228. [CrossRef]

29. Meng, J.G.; Zhang, X.D.; Tan, S.K.; Zhao, K.X.; Yang, Z.M. Genome-wide identification of Cd-responsive NRAMP transporter genes and analyzing expression of NRAMP 1 mediated by miR167 in Brassica napus. BioMetals 2017, 30, 917-931. [CrossRef]

30. Wang, C.; Chen, X.; Yao, Q.; Long, D.; Fan, X.; Kang, H.; Zeng, J.; Sha, L.; Zhang, H.; Zhou, Y.; et al. Overexpression of TtNRAMP6 enhances the accumulation of Cd in Arabidopsis. Gene 2019, 696, 225-232. [CrossRef]

31. Williams, L.E.; Pittman, J.K.; Hall, J. Emerging mechanisms for heavy metal transport in plants. Biochim. Biophys. Acta Biomembr. 2000, 1465, 104-126. [CrossRef]

32. Tiwari, M.; Sharma, D.; Dwivedi, S.; Singh, M.; Tripathi, R.D.; Trivedi, P.K. Expression in Arabidopsis and cellular localization reveal involvement of rice NRAMP, OsNRAMP1, in arsenic transport and tolerance. Plant Cell Environ. 2014, 37, 140-152. [CrossRef] [PubMed]

33. Li, J.; Wang, Y.; Zheng, L.; Li, Y.; Zhou, X.; Li, J.; Gu, D.; Xu, E.; Lu, Y.; Chen, X.; et al. The Intracellular Transporter AtNRAMP6 Is Involved in Fe Homeostasis in Arabidopsis. Front. Plant Sci. 2019, 10, 1124. [CrossRef] [PubMed]

34. Castaings, L.; Caquot, A.; Loubet, S.; Curie, C. The high-affinity metal Transporters NRAMP1 and IRT1 Team up to Take up Iron under Sufficient Metal Provision. Sci. Rep. 2016, 6, 37222. [CrossRef] [PubMed]

35. Zhang, J.; Zhang, M.; Song, H.; Zhao, J.; Shabala, S.; Tian, S.; Yang, X. A novel plasma membrane-based NRAMP transporter contributes to $\mathrm{Cd}$ and $\mathrm{Zn}$ hyperaccumulation in Sedum alfredii Hance. Environ. Exp. Bot. 2020, 176, 104121. [CrossRef]

36. Lanquar, V.; Lelièvre, F.; Bolte, S.; Hamès, C.; Alcon, C.; Neumann, D.; Vansuyt, G.; Curie, C.; Schröder, A.; Krämer, U.; et al. Mobilization of vacuolar iron by AtNRAMP3 and AtNRAMP4 is essential for seed germination on low iron. EMBO J. 2005, 24, 4041-4051. [CrossRef]

37. Mary, V.; Ramos, M.S.; Gillet, C.; Socha, A.L.; Giraudat, J.; Agorio, A.; Merlot, S.; Clairet, C.; Kim, S.A.; Punshon, T.; et al. Bypassing Iron Storage in Endodermal Vacuoles Rescues the Iron Mobilization Defect in the natural resistance associated-macrophage protein3natural resistance associated-macrophage protein4 Double Mutant. Plant Physiol. 2015, 169, 748-759. [CrossRef]

38. Wang, S.; Sun, J.; Li, S.; Lu, K.; Meng, H.; Xiao, Z.; Zhang, Z.; Li, J.; Luo, F.; Li, N. Physiological, genomic and transcriptomic comparison of two Brassica napus cultivars with contrasting cadmium tolerance. Plant Soil 2019, 441, 71-87. [CrossRef]

39. Xia, J.; Yamaji, N.; Kasai, T.; Ma, J.F. Plasma membrane-localized transporter for aluminum in rice. Proc. Natl. Acad. Sci. USA 2010, 107, 18381-18385. [CrossRef]

40. Bereczky, Z.; Wang, H.-Y.; Schubert, V.; Ganal, M.; Bauer, P. Differential Regulation of nramp and irt Metal Transporter Genes in Wild Type and Iron Uptake Mutants of Tomato. J. Biol. Chem. 2003, 278, 24697-24704. [CrossRef]

41. Li, Y.; Qin, Y.; Xu, W.; Chai, Y.; Li, T.; Zhang, C.; Yang, M.; He, Z.; Feng, D. Differences of Cd uptake and expression of MT family genes and NRAMP2 in two varieties of ryegrasses. Environ. Sci. Pollut. Res. 2018, 26, 13738-13745. [CrossRef]

42. Thomine, S.; Lelievre, F.; Debarbieux, E.; Schroeder, J.I.; Barbier-Brygoo, H. AtNRAMP3, a multispecific vacuolar metal transporter involved in plant responses to iron deficiency. Plant J. 2003, 34, 685-695. [CrossRef]

43. Kumar, S.; Stecher, G.; Tamura, K. MEGA7: Molecular Evolutionary Genetics Analysis Version 7.0 for Bigger Datasets. Mol. Biol. Evol. 2016, 33, 1870-1874. [CrossRef]

44. Qiao, X.; Li, Q.; Yin, H.; Qi, K.; Li, L.; Wang, R.; Zhang, S.; Paterson, A.H. Gene duplication and evolution in recurring polyploidization-diploidization cycles in plants. Genome Biol. 2019, 20, 38. [CrossRef] [PubMed]

45. Chen, C.; Chen, H.; Zhang, Y.; Thomas, H.R.; Frank, M.H.; He, Y.; Xia, R. TBtools: An Integrative Toolkit Developed for Interactive Analyses of Big Biological Data. Mol. Plant 2020, 13, 1194-1202. [CrossRef] [PubMed]

46. Duan, Y.; Zhu, X.; Shen, J.; Xing, H.; Zou, Z.; Ma, Y.; Wang, Y.; Fang, W. Genome-wide identification, characterization and expression analysis of the amino acid permease gene family in tea plants (Camellia sinensis). Genomics 2020, 112, 2866-2874. [CrossRef] 
47. Shen, J.; Zou, Z.; Xing, H.; Duan, Y.; Zhu, X.; Ma, Y.; Wang, Y.; Fang, W. Genome-Wide Analysis Reveals Stress and Hormone Responsive Patterns of JAZ Family Genes in Camellia Sinensis. Int. J. Mol. Sci. 2020, 21, 2433. [CrossRef] [PubMed]

48. Pfaffl, M.W. A new mathematical model for relative quantification in real-time RT-PCR. Nucleic Acids Res. 2001, 29, e45. [CrossRef]

49. Nelson, B.K.; Cai, X.; Nebenführ, A. A multicolored set of in vivo organelle markers for co-localization studies in Arabidopsis and other plants. Plant J. 2007, 51, 1126-1136. [CrossRef] [PubMed]

50. Han, Z.; Ma, Y.; Zhao, Y.; Qin, H.; Li, J.; Zhou, L.; Zhu, X.; Wang, Y.; Fang, W. Identification analysis of Plantacyanin (PLC) genes in Camellia sinensis and functional identification of CsPLC-3 in yeast. J. Hortic. Sci. Biotechnol. 2020, 95, 578-589. [CrossRef] 